\title{
Small-Scale Robot Formation Movement Using a Simple On-Board Relative Positioning System
}

\author{
Jim Pugh and Alcherio Martinoli \\ Swarm-Intelligent Systems Group \\ Ecole Polytechnique Fédérale de Lausanne, Switzerland \\ Email: \{jim.pugh,alcherio.martinoli\}@epfl.ch
}

Summary. We explore the problem of small-scale multi-robot formation control using only an unsophisticated on-board relative positioning module. The robotic setup and module are implemented and tested in a realistic virtual simulator, using standard formation types and algorithms, with and without radio communication. The experiments and results are then replicated using the real robotic platform. Finally, the simulation environment is used to assess the impact of potential improvements in the relative positioning module, and the significance and outlook of the system are discussed.

\section{Introduction}

Collective movement is an important aspect of many multi-robot tasks. With limited sensor range, it is often vital for robots to maintain close proximity to teammates within the group while moving within the environment. One specific case of collective movement is formation movement, where robots are required to keep a fixed distance and angle relative to other robots in the group. Formation movement is important for, among other things, control of Unmanned Air Vehicles (UAVs), collective transport of heavy/large objects, maintaining safe positions in an automated traffic system, and for guaranteeing good sensor coverage for tasks such as search and rescue missions.

Maintaining a formation requires that robots be given some positioning information about themselves and/or other robots. Artificial positioning systems can be roughly classified into two main categories: absolute and relative positioning systems. Absolute localization determines position in a global coordinate framework, while relative positioning only provides knowledge about the location of other robots relative to that of the sensing robot. For maintaining a formation, only relative distance and bearing information is needed, and although this information could also be obtained with an absolute positioning system and an effective communication channel, the communication overhead resulting from this solution might be much higher and less scalable than a solution based on a relative localization system. Also, certain situations do not allow for effective global localization (e.g., a distributed system in an unknown environment in which no GPS reception is possible). Currently, very 
few relative localization systems exist that can accomplish accurate and fully scalable performance and even fewer that rely only on unsophisticated hardware.

Section 2 of this paper provides some background on robotic formation movement and relative positioning between mobile robots. Section 3 describes the equipment and formation techniques used. Experiments are described in Section 4 and results are given in Section 5. Section 6 discusses the implications of the results the outlook for the system. Section 7 concludes the paper.

\section{Background}

There has been a substantial amount of research on robotic formation movement in recent years. In [1], motor schemas were used in simulation and with Nomad 150 robots to allow two robots to navigate in formation while avoiding obstacles. [6] developed an algorithm for formation movement and implemented it in simulation as well as with four real Pioneer2 robots in a variety of different dynamic formations. In [13], several formation control strategies were proposed, analyzed, and tested on a group of three robots. An algorithm for formation movement planning was developed and analyzed in [9] and tested on three Magellan Pro robots. Formation movement in simulation only has also been explored with a variety of approaches (e.g., [2], [4], [5], [20]).

There has been relatively little work done on development of on-board relative positioning systems for small-scale robots. A system using ultrasound and radio with time-of-flight for calculating range but no bearing between robots was described in [8]. In [3], an ultrasonic/radio system which calculated both range and bearing was presented and characterized. [12] and [17] described and characterized an infraredbased system which calculated range and bearing using the Received Signal Strength Indication (RSSI). Another infrared system for range and bearing was mentioned in [15]. On-board relative localization has been done using more complicated devices (e.g., laser range finders and cameras) on several occasions (e.g., [7], [11], [18]).

Of all the previous studies on robotic formation movement, almost all simulated relative positioning within the formation using some sort of global positioning system. Only [6] used an on-board relative positioning system, and this system used both a laser range-finder and a mobile camera, and would therefore be infeasible to use on low-cost, miniature robots. It remains to be tested whether a simple on-board relative positioning system can effectively allow formation movement with real robots.

\section{Equipment and Algorithms}

The system we have used for our formation experiments is a small-scale robotic platform (Moorebots, see [19]). These robots are roughly cylindrical, with a 24 $\mathrm{cm}$ diameter. However, nothing prevents a further miniaturization of the modules presented so that the same localization techniques could be used on robots of a few $\mathrm{cm}$ in diameter. The robots used are equipped with a PC/104 stack with IEEE 
802.11 wireless capability and an Arcom Viper processor board running a low-power $400 \mathrm{MHz}$ Intel ${ }^{\circledR}$ PXA255 XScale; this configuration allows us to achieve 4 hours of autonomy. All experiments were performed in an arena $3.4 \times 3.4 \mathrm{~m}$. The relative localization system is an on-board module which allows the robots to determine the range and bearing of other nearby robots based on the strength of a modulated infrared signal, and serves simultaneously as a local communication channel with low bit rate (approximately $16 \mathrm{bits} / \mathrm{s}$ ) [12]. The system can also be used to communicate at a significantly higher bit rate if the relative positioning aspect is disabled.

The relative localization and communication module used for formation control is built entirely from off-the-shelf components [12]. It is mounted on the PC/104 stack which is off-center on the Moorebot platform (see Fig. 1). Signals are sent with infrared (IR) light and modulated/demodulated using RF technology. The system is controlled by a dedicated on-board PIC microcontroller. Each module has twelve IR Light Emitting Diodes (LEDs) arranged into four independently controllable clusters at $90^{\circ}$ spacing for full $360^{\circ}$ angular coverage. The range of the module goes up to $310 \mathrm{~cm}$. Each module has four photodiode receivers at $90^{\circ}$ spacing. Signal strengths at these receivers allow for calculation of the relative distance and angle to other robots. At distances less than $30 \mathrm{~cm}$, signal strengths become distorted by optical effects, making accurate calculation impossible. Range is determined by the Received Signal Strength Indication (RSSI). The RSSI of the four receivers can be compared to calculate the direction of the other robot. The intensity of the received signal decays asymptotically as the range between robots increases. The module currently operates at $2 \mathrm{~Hz}$, which allows a group of up to 10 robots to all exchange their relative positions concurrently. Three different transmission power levels are possible, hence the operational range can be lessened to decrease interference between densely packed robots. The accuracy of the relative localization module was analyzed in [17]. The average error in bearing is $6.10^{\circ}$ and the average error in position is $2.77 \mathrm{~cm}$ at the closest range of $30 \mathrm{~cm}$ range and $17.70 \mathrm{~cm}$ at a maximum range of $300 \mathrm{~cm}$.

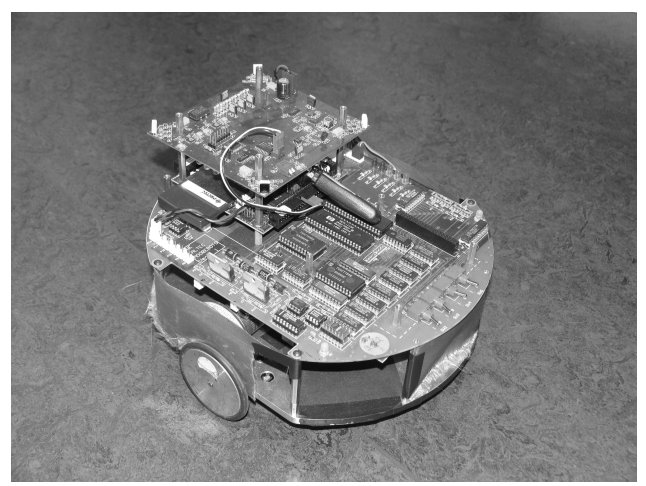

Fig. 1. The Moorebot robot with on-board relative positioning module 
By itself, the relative localization system can only allow some robot A to detect the range and bearing of another robot $\mathrm{B}$, not the relative direction in which $\mathrm{B}$ is facing. This limitation can be overcome if we introduce high-speed communication between the robots. The relative positioning observation of robot $\mathrm{B}$ can be sent to robot A, which will allow A to determine B's heading. The data rate of the current relative localization system's communication is not sufficient to accomplish this, but a faster communication channel could. In our case, the on-board IEEE 802.11 wireless interface is sufficient for this purpose.

For our formation control algorithm, we use the approach described in [1], with potential field attraction/repulsion for maintaining formation position, combined with obstacle avoidance using a motor schema method.

We consider two different types of robot formations: location-based and headingbased. In location-based formations, we care about robot positions in the formation and ignore the different directions which robots may be facing. In heading-based formations, robots are required to maintain a certain position and heading for the formation to be correct. We consider the further subcategory of leader headingbased formations, where follower robot locations are determined by the position and heading of the leader, but the headings of the followers are ignored. It is only possible to implement heading-based formations with robots that can detect the headings of neighboring robots, making them more difficult to maintain.

\section{Experimental Setup}

Four robots are placed in a diamond shape in an arena measuring $3.4 \mathrm{~m} \mathrm{x} 3.4 \mathrm{~m}$ in both simulation and with real robots. All robots are offset $0.4 \mathrm{~m}$ from the center of the formation, and the leader robot (the one at the front of the formation) travels an elongated figure 8 path around the arena (see Fig. 2). For simulation, we use the realistic robotic simulator Webots [16]. With the real Moorebots, we use an overhead camera to track the location and bearing of the robots; the system has an error of up to $1 \mathrm{~cm}$ in position and 1 degree in angle. A close-up of the real robot formation is shown in Fig. 3. Robots operate in three different modes:

Mode 1: No Relative Positioning - The follower robots are preprogrammed with the course they must take to maintain formation with the leader robot. There is no feedback to compensate for robot drift due to wheel slip or obstacle avoidance.

Mode 2: Relative Positioning Without Communication - The on-board relative positioning module is used to detect the range and bearing of other robots in the formation. Position is maintained by using a potential field approach to keep the proper distance to all other robots. This mode is most effective in maintaining a location-based formation.

Mode 3: Relative Positioning With Communication - The on-board relative positioning module is used along with wireless communication to allow robots to detect the range, bearing, and heading of other robots. Position is maintained by using the potential field approach to move to the proper position based on the location and heading of the leader; the system therefore attempts to maintain a leader heading- 
based formation.

Comparing to previous formation-maintaining techniques, the methods used in Mode 2 and Mode 3 are roughly the equivalent of Unit-center-referenced and Leaderreferenced methods described in [1], respectively.

Our first experiment compared Mode 1 and Mode 2 running with a locationbased formation, and our second compared Mode 2 and Mode 3 running with a leader heading-based formation, both in simulation and with real robots. Mode 2 is well-suited for location-based formation, since it maintains the proper distances between teammates, but not for leader heading-based formation, since it ignores the heading of the leader; Mode 3 is well-suited for leader heading-based formation, since it takes the leader heading into account. Mode 2 and Mode 3 were run again in simulation with a $16 \mathrm{~Hz}$ relative positioning update rate instead of $2 \mathrm{~Hz}$; this is a realistic rate to expect from future infrared relative positioning systems and allows us to explore what impact these systems might have on formation control. The leader robot moves at $10 \mathrm{~cm} / \mathrm{s}$ (as compared to $2 \mathrm{~cm} / \mathrm{s}$ with the much larger Pioneer 2 robot in [6]). Experiments were run for 120 seconds.

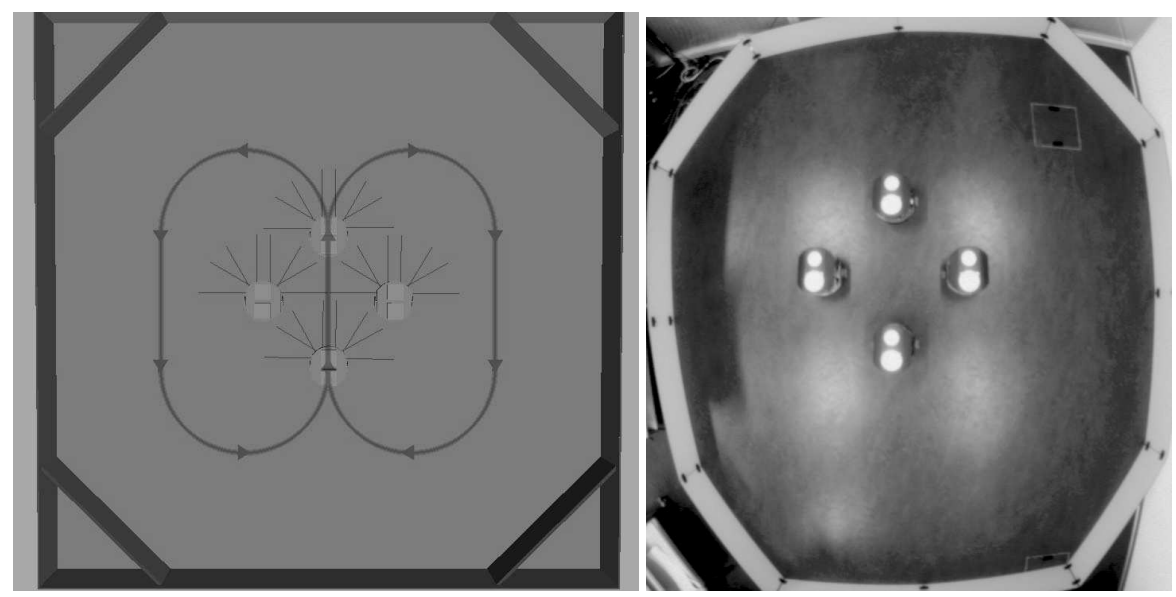

Fig. 2. (a) Robotic arena in Webots, our realistic robotic simulator. The path of the formation leader is shown. Lines protruding from Moorebots represent proximity sensors. (b) Picture of real arena taken by overhead tracking camera with Moorebots in initial formation positions.

\section{Results}

Comparison between Mode 1 and Mode 2 for location-based formation can be seen in Fig. 4a for simulation and Fig. $4 \mathrm{~b}$ for real robots. The error given here is the average error in distance between robots in the formation. We see very similar performance between the simulated environment and the real-world one. Initially, Mode 1 is able 


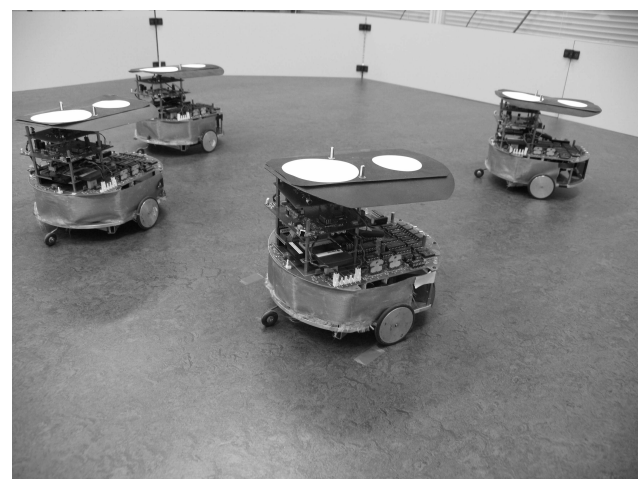

Fig. 3. Formation of Moorebots

to maintain a better formation than Mode 2, since the error due to obstacle avoidance and slip noise accumulates over time, but it very quickly surpasses the error of Mode 2, which remains approximately constant throughout the experiment. This demonstrates the the on-board relative positioning module allows the robots to move about in formation.
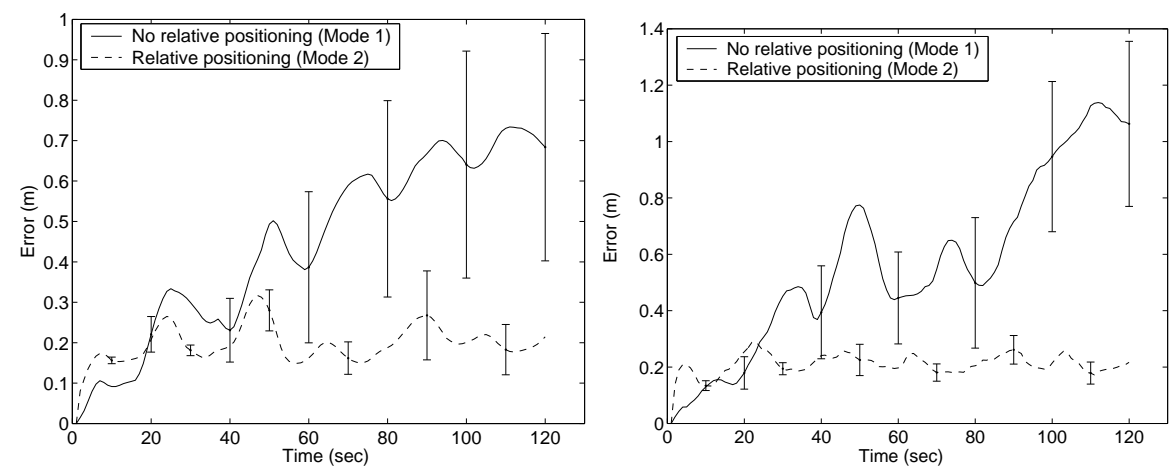

Fig. 4. Average error in position for location-based formation over (a) 100 runs in simulation and (b) 10 runs with real robots. Error bars represent standard deviation over runs.

Comparisons between Mode 2 and Mode 3 for leader heading-based formation can be been in Fig. 5a for simulation and Fig. 5b for real robots. There is again very close matching between simulated and real-world results. The error for Mode 2 is significantly higher for leader heading-based formation than for location-based formation, as the robots are unable to detect the heading of the leader robot. Mode 3 is able to achieve slightly better performance than Mode 2, but not significantly so. This is likely due to a combination of several factors. When the leader robot turns, robots on the outside of the formation must move much faster in order to keep their proper position in the leader heading-based formation; this isn't the case in a location-based 
formation, where followers can simply mimic the motion of the leader to keep the formation shape. In the Mode 3 formation algorithm, follower robots determine their proper positions from the leader robot only, not all other robots as in Mode 2; this amplifies the error caused by noise in the relative positioning measurements and prevents follower robots from having any knowledge of their proper position if the leader robot's relative positioning signal is occluded by some other follower robot. The effect of both of these factors is amplified by the slow update rate $(2 \mathrm{~Hz})$ of the relative positioning system. There can potentially be a 0.5 second delay between receiving a relative positioning signal and the robot being able to process the signal, and new signals are only received every 0.5 seconds. We suspect that all these factors prevent the addition of communication from significantly improving the performance on leader heading-based formation movement.
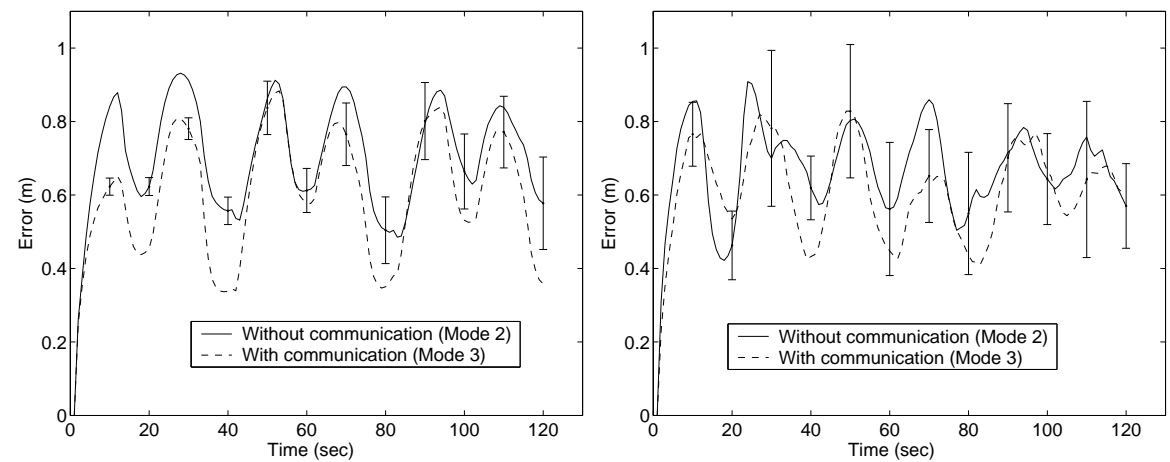

Fig. 5. Average error in position for leader heading-based formation over (a) 100 runs in simulation and (b) 10 runs with real robots. Error bars represent standard deviation over runs.

Comparisons between standard setup and rapid relative positioning (where the update rate is increased from $2 \mathrm{~Hz}$ to $16 \mathrm{~Hz}$ ) can be seen in Fig. $6 \mathrm{a}$ and Fig. $6 \mathrm{~b}$ for Mode 2 and Mode 3, respectively. We see that in Mode 2, position error decreases and remains more constant with the rapid update rate. The effect in Mode 3 is much more drastic; error decreases by nearly a factor of ten and consistently remains low, suggesting that the difficulties encountered in this mode can be eliminated by using a relative positioning system with a faster update rate. The credibility of these results is strengthened by the close matching between simulation and real experiments previously shown.

\section{Discussion and Outlook}

The impact of the update rate of robot positioning for formation control has not been mentioned in previous work, likely because of the lack of experiments using real on-board relative positioning systems for formation movement. If a very accurate 

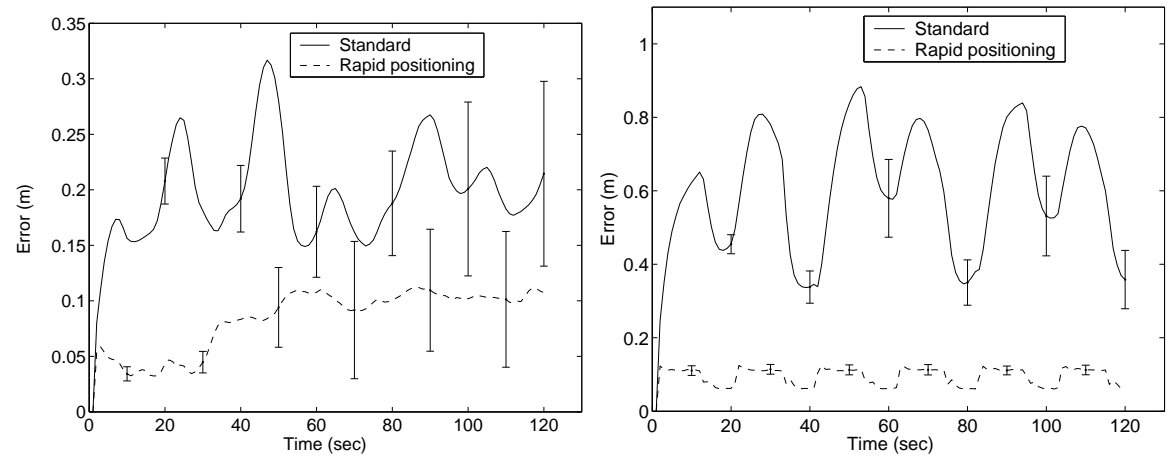

Fig. 6. Average error in position over 100 runs in Webots for (a) location-based formation with Mode 2 and (b) leader heading-based formation with Mode 3 comparing standard and rapid update rates. Error bars represent standard deviation over runs.

formation is to be maintained, the only way to compensate for a slow update rate would be to limit the robots' movement rate. Although it is not mentioned in [6], we suspect that the slow robot speed $(20 \mathrm{~mm} / \mathrm{s})$ was at least partially due to a slow update rate of the relative positioning used.

The on-board relative positioning system we use here is only a first version of the concept; with redesigns and improvements in technology it should be possible to make new simple systems of the same type with faster update rates, longer ranges, and improved communication abilities. It may be that the communication capabilities of future versions will be fast enough that wireless radio is no longer required to allow robots to determine the headings of other robots. However, radio may still offer advantages for more sophisticated algorithms with more required communication.

Using infrared as a medium for sending relative positioning signals has various costs and benefits as opposed to other popular methods. Infrared signals have the potential to be very fast, with the signally duration potentially being on the order of microseconds, which could allow for very fast update rates and fast communication. Infrared transceivers could also be produced at a very small scale, allowing the same concepts to be used for future micro robots. However, using RSSI to calculate range and bearing leaves the system susceptible to interference from external signals, which might limit the accuracy. The emitters and receivers are also very sensitive to miscalibration for the same reason. Finally, infrared light can be occluded if a robot or some other obstacle gets in the way, which could prevent the signal from accurately reaching other robots.

Ultrasound signals synchronized with radio transmissions is the other most popular current method of relative positioning along with infrared. Using time-of-flight for positioning can be very accurate, especially over longer ranges; in [3], error on the order of only a few millimeters was present with ranges up to $810 \mathrm{~cm}$. Because the received signal strength is no longer used to calculate range and bearing, the transceivers do not need to be carefully calibrated for the system to work. Ultrasound is also much better able to pass through solid objects than infrared, making ultra- 
sound systems less sensitive to occlusion. A potential problem with ultrasound is that a signal is slow to dissipate, especially in an enclosed environment. This could limit the rate at which robots could transmit signals, especially as the number of robots present grows in number. "Crosstalk" between ultrasonic sensors and reflections in a known problem for proximity sensing, and this effect caused problems with the system used in [8]. Ultrasonic transceivers also tend to be larger than infrared ones, which can make it more difficult to adapt them for use with very small robots.

A recent method of positioning which has not yet been adapted for use with mobile robots is using radio signals with time-of-flight and phased array reception. By sending radio messages back and forth and accurately measuring the times of transmission and arrival, it is possible to measure the range between robots (for example, see [14]), and bearing can be calculated by comparing the phases of received signals on multiple receivers (see [10] for example). Radio localization can be used over much longer ranges than infrared or ultrasound, is not sensitive to miscalibration since it uses time-of-flight, and is not very sensitive to occlusion. However, it is difficult to very accurately measure signal arrival times, and therefore current radio localization techniques tend to have errors on the order of several tens of centimeters. This is too large for small-scale robot systems and certainly too large for micro robots. Future improvements in the technology may allow this problem to be overcome.

\section{Conclusion}

We have shown how a simple on-board relative positioning module can be used to accomplish effective formation control in small-scale multi-robot systems. Experiments were run in simulation and validated using real robots. The impact of improving the update rate of the module was explored in simulation. Significance of the results and the outlook of the system were discussed.

\section{Acknowledgements}

Jim Pugh and Alcherio Martinoli are currently sponsored by a Swiss NSF grant (contract Nr. PP002-68647).

\section{References}

1. Balch, T. \& Arkin, T. C., (1998) "Behavior-Based Formation Control for Multirobots Teams”, IEEE Trans. on Robotics and Automation, Vol. 14, No. 6, pp. 926-939.

2. Balch, T. \& Hybinette, M. (2000) "Social Potentials for Scalable Multi-Robot Formations", Proc. of the IEEE International Conference on Robotics and Automation, San Francisco, CA, April, pp. 73-80.

3. Bisson, J., Michaud, F. \& Létourneau, D. (2003) "Relative Positioning of Mobile Robots Using Ultrasounds", IEEE Int. Conf. on Robotics and Authomation IROS03, Oct, Las Vegas, Nevada, USA, pp. 1783-1788. 
4. Chen, Q. \& Luh, J. Y. S. (1994) "Coordination and Control of a Group of Small Mobile Robots", Proc. of the IEEE International Conference on Robotics and Automation, San Diego, CA, pp. 23152320.

5. Desai, J. P., Ostrowski, J. P., \& Kumar, V. (2001) "Modeling and Control of Formations of Nonholonomic Mobile Robots", IEEE Trans. on Robotics and Automation, Vol. 17, No. 4, pp. 905-908.

6. Fredslund, J. \& Matarić, M. J. (2002) "General Algorithm for Robot Formations Using Local Sensing and Minimal Communication", Special Issue on Advances in Multi-Robot Systems, Arai T., Pagello E., and Parker L. E., editors, IEEE Trans. on Robotics and Automation, Vol. 18, No. 5, pp. 837-846.

7. Fox, D. et al (2000) "General Algorithm for Robot Formations Using Local Sensing and Minimal Communication”, Autonomous Robots, Vol. 8, No. 3, pp. 325-344.

8. Grabowski, R. \& Khosla, P. (2001) "Localization Techniques for a Team of Small Robots", Proc. of the IEEE/RSJ International Conference on Intelligent Robots and Systems, Maui, HI, Oct 29-Nov 3, pp. 1067-1072.

9. Hao, T. et al (2003) "Planning and Control of UGV Formations in a Dynamic Environment: A Practical Framework with Experiments", Proc. of the IEEE International Conference on Robotics and Automation, Taipei, Taiwan, Sept 14-19, pp. 1209-1214.

10. Hashemi, H., Guan, X. \& Hajimiri, A. (2004) "A Fully Integrated 24 GHz 8-path PhasedArray Receiver in Silicon", IEEE Int. Solid-State Circuits Conf. Dig. Tech. Papers, Feb., pp. 318-319.

11. Howard, A., Matarić, M. J., \& Sukhatme, G. S. (2003) "Localization for Mobile Robot Teams: A Distributed MLE Approach”, Springer Tracts in Advanced Robotics, Vol. 5, Jan 2003, pp. 146-155

12. Kelly, I. D. \& Martinoli, A. (2004) "A Scalable, On-Board Localisation and Communication System for Indoor Multi-Robot Experiments”, Special Issue on Sensor Simulation and Smart Sensors, C. Loughlin, editor, Sensor Review, Vol. 24, No. 2, pp. 167-180.

13. Lawton, J. R. T., Beard, R. W., \& Young, B. J. (2003) "A Decentralized Approach to Formation Maneuvers", IEEE Trans. on Robotics and Automation, Vol. 19, No. 6, pp. 933-941.

14. Lee, J. Y. \& Scholtz, R. A. (2002) "Ranging in a Dense Multipath Environment Using an UWB Radio Link", IEEE Journal on Selected Areas in Communications, Vol. 20, No. 9, pp. 1677-1683.

15. McLurkin, J. \& Smith, J. (2004) "Distributed Algorithms for Dispersion in Indoor Environments using a Swarm of Autonomous Mobile Robots", Proc. of the Seventh Int. Symp. on Distributed Autonomous Robotic Systems, Toulouse, France, June 23-25, pp. 381-390.

16. Michel, O. (2004) "Webots: Professional Mobile Robot Simulator", Int. J. of Advanced Robotic Systems, Vol. 1, pp. 39-42.

17. Pugh, J. \& Martinoli, A. (2006) "Relative Localization and Communication Module for Small-Scale Multi-Robot Systems", Proc. of the IEEE International Conference on Robotics and Automation, Miami, FL, May 15-19, pp. 188-193.

18. Rekleitis, I., Dudek, G. \& Milios, E. (2001) "Multi-robot collaboration for robust exploration", Annals of Mathematics and Artificial Intelligence, Vol. 31, pp. 7-40.

19. Winfield, A.F.T. \& Holland, O.E. (2000) "The application of wireless local area network technology to the control of mobile robots", Microprocessors and Microsystems, Vol. 23, pp. 597-607.

20. Yamaguchi, H., Arai, T. \& Beni, G. (1997) " A distributed control scheme for multiple robotic vehicles to make group formations", Robotics and Autonomous Systems, Vol. 36, No. 4, pp. 125-147. 